\title{
Korrespondenzen.
}

Aus der Anatomischen Anstalt der Universität in Berlin.

\section{Ueber Ursache und Bedingung, Naturgesetz und Regel.} Von R. Fick.

Unser verehrter Meister der Entwicklungsmechanik R o u x hat am Schlusse seines obgenannten Aufsatzes in dieser Wochenschrift (1922 Nr. 37) gegen meine Ausführungen über denselben Gegenstand in einer Weise Stellung genommen, die mich zur Abwehr zwingt. Ich bin dazu um so mehr verpflichtet, da Roux nicht anführte, wo ich die von ihm befehdeten Ausführungen veröffentlicht habe, soda $\beta$ die Leser dieser Zeitschrift nicht vergleichen können, was ich in Wirklichkeit gesagt habe, und weil mir auch sachlich die $A b$ weisung der Vorschläge $R$ oux' richtig scheint.

Roux behauptet, ich hätte die von ihm "hier reproduzierte scharfe Sonderung von Naturgesetz und Naturregel für überflüssig und als gegen den Sprachgebrauch verstoßend verworfen". Er sagt weiterhin, ,ob diese Sonderung volkstümlich wird, ist für die Wissenschaft unerheblich. Die jetzige Opposition ist nur der Ausdruck des üblichen Widerstandes des Alten, Herkömmlichen gegen den Fortschritt".

Demgegenüber möchte ich feststellen, daß ich in meiner Mitteilung (Sitzber. d. Preuß. Akademie d. Wiss., 15. VII. 1920, 14, 1921) durchaus nicht behauptet habe, daß Roux' Streben nach scharfer Sonderung der Ausdrücke überflüssig sei, und ebensowenig behauptet habe, die Sonderung müsse volkstümlich sein. In Wahrheit habe ich gerade das Gegenteil davon getan, nämlich gesagt, dip Ausdrücke "Gesetz" und "Regel" seien $z$ u volkstümlich, um in der Wissenschaft als scharfbegrenzte Begriffsbezeichnungen brauchbar sein zu können. Ich bin durchaus der Meinung, daB es sehr vorteilhaft und wünschenswert wäre, wenn die von Roux besprochenen Begriffe in der Wissenschaft scharf auseitiandergehalten würden, aber das wäre nur möglich, wenn neue Bezeichnungen dafür eingeführt würden, nicht etwa durch künstliche Bedeutungsbeschränkung der alten, nun einmal unscharf gebrauchten Volkswörter. Ich schloB meine Ausführungen wörtlich: „Die angezogenen Beispiele geben ein Bild von der Unschärfe der Ausdrücke: Gesetz und Regel, Ursache, Vorbedingung und Veranlasssung. Und ich glaube, daB es eine Unmöglichkeit bleiben wird, dem wissenschaftlichen Gebrauch dieser Wörter bestimmte Fesseln anzulegen und jedem von ihnen eine scharf bestimmte Bedeutung für den wissenschaftlichen Gebrauch aufzuzwingen. Das ist leicht möglich für ein in die Wissen: schaft neu eingeführtes Wort, wie für den Namen einer bestimmten Krankheit oder eine bestimmte Gruppe van Krankheitszeichen, es ist aber eben unmöglich bei. Wörtern, die im Sprachgebrauch der weitesten Allgemeinheit stehen. Solche Wörter des allgemeinen Sprachschatzes lassen sich nicht (für den wissenschaftlichen Gebrauch) mit künstlichen Stachelżäunen umgeben. Trotzdem scheint mir die genaue, seit Jahrzehnten fartgesetzte klärende Erörterung der besprochenen Begriffe, an der sich W. Roux mehrfach hervorragend beteiligt hat, ke ineswegs müig und unf ruchtbar, auch wenn sich schlieBlich herausgestellt hat, daB es durch die Natur der Sache einfach unzulässig und unmöglich bleiben wird. für die wissenschaftliche Anwendung dieser Ausdrücke starre Regeln durchzusetzen."

Berlin, 29. I. 1923. 